\title{
Effects of linseed consumption for a short period of time on lipid profile and atherosclerotic lesions in rabbits fed a hypercholesterolaemic diet
}

\author{
Camila Rodrigues Prim, Liz Andréa Villela Baroncini*, Leonardo Brandão Précoma, Pedro Henrique \\ Lamach Caron, Guilherme Winter, Mônica Olímpia Dall'Oglio Poletti and Dalton Bertolim Précoma \\ Center of Health and Biological Sciences, Pontificia Universidade Católica do Paraná, Rua Imaculada Conceição, 1155 , \\ Prado Velho, CEP 80215-901 Curitiba, Paraná, Brazil
}

(Received 5 January 2011 - Revised 9 May 2011 - Accepted 31 May 2011 - First published online 27 July 2011)

\begin{abstract}
Linseed contains biologically active substances, such as lignans, fibres and linoleic acid, which are believed to provide cardioprotective effects. The objective of the present study was to assess the potential hypolipaemic, anti-atherogenic and anti-inflammatory effects of linseed consumption using an experimental animal model, with rabbits fed a hypercholesterolaemic diet ( $1 \%$ cholesterol extracted from lyophilised egg). A total of twenty white male rabbits were selected and divided into two groups: group I (GI), control group, ten rabbits; group II (GII), ten rabbits. The animals were fed a hypercholesterolaemic diet for $56 \mathrm{~d}$. For the GII diet, ground linseed was added from day 29 through to day 56. Animals underwent aortic arch and descending aorta dissection on day 56 for histological, morphometric and immunohistochemical analysis. At the end of the experiment, GII animals presented with lower levels of total cholesterol (TC, $10068 \cdot 3$ v. $16767.0 \mathrm{mg} / \mathrm{l} ; P<0.05$ ) and lower levels of LDL-cholesterol (LDL-C; $10743.2 v .15961 .2 \mathrm{mg} / \mathrm{l} ; P<0.05$ ) when compared with the GI control group. There was no significant difference in serum HDL-cholesterol and TAG between the two groups. Almost all animals exhibited type III atherosclerotic lesions in the descending aorta. There was no statistically significant difference between the intima area and the intima:media layer area ratio in both groups. There was no difference between the positive areas for vascular cell adhesion molecule-1 and intercellular adhesion molecule-1 molecules between the groups. Linseed consumption showed hypolipaemic action by reducing LDL-C and TC levels; however, this cholesterol-lowering effect did not reduce the atherosclerotic lesions induced by a hypercholesterolaemic diet ( $1 \%$ cholesterol) for a short period of time.
\end{abstract}

Key words: Linseed: Atherosclerosis: Hypercholesterolaemia

Epidemiological studies have demonstrated a positive correlation between exposure to risk factors such as obesity, dyslipidaemia, smoking, physical inactivity and insulin resistance and the progression of atherosclerosis ${ }^{(1)}$. Fornés et $a l^{(2)}$ added that eating habits are risk factors for CVD, in that high intakes of cholesterol, fat and SFA along with low fibre intake, participate in the aetiology of dyslipidaemia. Clinical evidence has emphasised that the adoption of preventive measures could contribute to minimising and preventing coronary events. Linseed contains biologically active substances such as lignans, fibre and linoleic acid that have cardioprotective effects. The lignan compounds, when ingested, are capable of promoting reductions in serum cholesterol because of their modulatory function through the cholesterol acyltransferase enzyme (acyl CoA) and 7- $\alpha$-hydroxylase involved in their metabolism. In randomised clinical trials that enrolled patients with CHD, $n-3$ fatty acid supplements significantly reduced cardiovascular events. $n-3$ Supplements can also slow the progression of atherosclerosis in $\mathrm{CHD}^{(3)}$.
The American Heart Association recommends eating plantderived $n$-3 fatty acids including flaxseeds and their oils ${ }^{(4)}$. The present study aimed to evaluate the potential lipid-lowering capacity, reversal of established atherosclerotic lesions and anti-inflammatory effects of linseed in an experimental model of rabbits fed a hypercholesterolaemic diet ( $1 \%$ cholesterol from lyophilised egg).

\begin{abstract}
Methods
Animals

A total of twenty white adult male rabbits (New Zealand), with a mean age of 4 months, were selected for the present study. The sample size was chosen on the basis of the studies of Prasad $^{(5,6)}$ and Bhathena et al. ${ }^{(7)}$, who used six to ten rabbits per experimental group. Animals were handled in compliance with the Guiding Principles in the Care and Use of Animals and protocol approval was obtained from the Pontifical
\end{abstract}

Abbreviations: GI, group I; GII, group II; HDL-C, HDL-cholesterol; LDL-C, LDL-cholesterol; TC, total cholesterol.

*Corresponding author: L. A. V. Baroncini, fax +554132711657, email lizandreabaroncini@hotmail.com 
Catholic University Animal Research Committee. The animals were divided into two groups: group I (GI), control group, ten rabbits; group II (GII), ten rabbits. During the $56 \mathrm{~d}$ study, the animals were fed a specific diet (Nuvilab ${ }^{\circledR}$; Colombo, Brazil) plus $1 \%$ cholesterol from lyophilised egg. For the GII diet, $8 \mathrm{~g} / \mathrm{kg}$ of ground linseed was added from day 29 through day 56 , totaling an average of about $24 \mathrm{~g}$ of linseed per $\mathrm{d}$ for each rabbit. There was no interference in the satiation of the animals regarding the specific diet (Nuvilab ${ }^{\circledR}$ plus cholesterol). On day 56, the animals were subjected to aortic arch and descending aorta dissection. Anaesthesia was induced with ketamine $\left(3.5 \mathrm{mg} / \mathrm{kg}\right.$; Vetanarcol ${ }^{\circledR}$; König, Avellaneda, Argentina) and intramuscular xylazine $\left(5 \mathrm{mg} / \mathrm{kg}\right.$; Coopazine ${ }^{\circledR}$; Coopers, São Paulo, Brazil). After the procedure, the rabbits were killed by a lethal barbiturate dose.

\section{Blood chemistry}

Blood samples were obtained on the first day of the experiment and also immediately before killing by cardiac puncture. Clinical laboratory assessment included total cholesterol (TC), HDL-cholesterol (HDL-C), LDL-cholesterol (LDL-C) and TAG. Measurements were taken using an automated system (Abbott Architect ci8200; Abbott Laboratories, Abbott Park, IL, USA).

\section{Histological analysis}

The arteries were removed and washed with 10\% formaldehyde buffered with phosphate $(\mathrm{pH} \mathrm{7.6)}$ and then paraffin embedded. For evaluation of this experimental model, qualitative and quantitative histological measurements were obtained. Haematoxylin and eosin-stained slices were analysed blindly in a five head Olympus ${ }^{\circledR}$ BX40 microscope (Olympus, Tokyo, Japan). The atherosclerotic lesions were graded from 0 to 6 , according to the qualitative criteria proposed by Stary et $a l{ }^{(8)}$. Morphometric analysis was performed on the slides stained with orcein (elastic) to determine the area of the intimal and medial layers of the aortic arch and descending aorta, as well as to determine the intima:media layer area ratio (the area of the intimal layer divided by the area of the medial layer). For this assessment, the more injured segment was previously selected from haematoxylin and eosin sections. The microscopic analyses were performed in conjunction with Image Pro-plus ${ }^{\circledR} 4.5$ software (Media Cybernetics Inc., Silver Spring, MD, USA).

\section{Immunohistochemistry}

We evaluated the concentration of vascular cell adhesion molecule-1 and intercellular adhesion molecule-1 in the intima of the arteries using primary monoclonal antibodies. Reading was performed with an Olympus ${ }^{\circledR}$ BX50 microscope (Olympus), with an objective of $20 \times$.

\section{Statistical analysis}

Categorical variables were expressed as percentages and continuous variables were expressed as mean and standard deviation values and median values. The Shapiro-Wilks test was used for evaluating sample normality and the Student's $t$ test and Mann-Whitney non-parametric test were used for quantitative parameters in the comparison between GI and GII. Statistical significance was indicated by a value of $P<0.05$.

\section{Results}

\section{Animal weights}

The initial body weight of animals in GI and GII were similar. At the time of the end of the study, GI animals exhibited a mean body weight of $2.98 \mathrm{~kg}$, whereas GII animals had a mean body weight of $2.72 \mathrm{~kg}$, with a weight change of 67.5 and $56.4 \%$, respectively. The GII had less weight gain when compared with the GI at different time periods $(P<0 \cdot 01)$ The average weights of animals measured at different time periods of the experimental period are summarised in Table 1.

\section{Lipid profiles}

Baseline TC, HDL-C and TAG levels were relatively equal in all groups before initiation of the diet. There was an increase in serum TC in both groups after the experimental period, corresponding to a percentage of variation of approximately $2379 \%$ in GI and $1249 \%$ in GII. The minimum, maximum, median and SD of TC for the animals are summarised in Table 2. Although the increase in TC occurred in both groups, this increase was statistically higher in the group without linseed in the diet $(P<0.046)$. Similar results were observed for LDL-C (Table 3). At the end of the experiment, the animals supplemented with linseed displayed lower values of LDL-C when compared with the control group $(P<0.05)$. Analysis of HDL-C and serum TG indicated no significant difference between the groups at the end of the experiment (data not shown)

\section{Histological analysis}

At the end of the experiment, it was observed that there was no statistically significant difference between animal groups with respect to the probability of type I, II, III, IV and V lesions, or the presence of any injuries. Almost all animals showed type III atherosclerotic lesions in the descending aorta (Fig. 1).

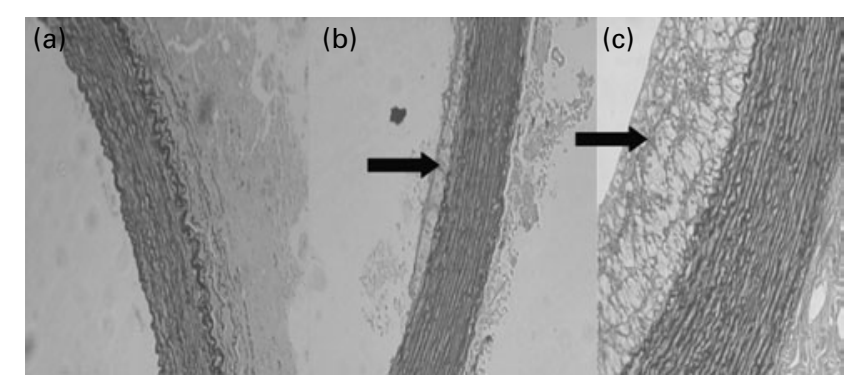

Fig. 1. Types of atherosclerotic lesions seen in the aortic arch. (a) Normal aortic arch; (b) type I atherosclerotic lesion; (c) type III atherosclerotic lesion. $\rightarrow$ Intima layer. 
Table 1. Animal weights at different time points

(Mean values and standard deviations, $n$ 10)

\begin{tabular}{llccc}
\hline Weight & Group* & Mean $(\mathrm{kg})$ & SD & $P \dagger$ \\
\hline Initial & GI & 1.78 & 0.15 & \\
& GII & 1.75 & 0.16 & 0.659 \\
$15 \mathrm{~d}$ & GI & 2.36 & 0.29 & \\
& GII & 2.08 & 0.19 & 0.021 \\
$22 \mathrm{~d}$ & GI & 2.58 & 0.33 & \\
& GII & 2.29 & 0.19 & 0.026 \\
Final & GI & 2.98 & 0.41 & \\
& GII & 2.72 & 0.19 & 0.096 \\
\hline
\end{tabular}

GI, group 1; GII, group II.

* Group GI, control group; group GIl, study group.

† Student's $t$ test.

\section{Morphometric analysis}

There was no statistically significant difference between the intimal area and the intima:media layer area ratio of both groups in the aortic arch and descending aorta.

\section{Immunohistochemistry}

Analysis of positive areas for vascular cell adhesion molecule1 and intercellular adhesion molecule- 1 showed no statistically significant differences in the segments of the aortic arch and descending aorta between GI and GII.

\section{Discussion}

In the present study, rabbits were fed $8 \mathrm{~g}$ linseed $/ \mathrm{kg}$ weight per $d$. Few previous studies ${ }^{(9)}$ have determined the safe and effective amount of linseed for rabbits, and despite it seeming excessive, the linseed amount we administered was well tolerated by the animals and effectively interfered in their lipid profile. It can be observed in the present study that there was an increase in TC and LDL-C in both groups, but at lower levels in the group with supplemental linseed. This potential reducing effect of linseed is noteworthy, because it is known that the reduction of $10 \mathrm{mg} / \mathrm{l}$ in LDL-C results in a decrease of approximately $1-2 \%$ in the relative risk for cardiovascular morbidity ${ }^{(10-14)}$. The mechanisms by which linseed acts to prevent CVD are not entirely clear. There are reports that the hypocholesterolaemic effects of linseed may be attributed to the functional components present in its composition of lignans, soluble fibres and linolenic $\operatorname{acid}^{(10)}$. Recent studies ${ }^{(15,16)}$ report that the lignans act positively in plasma lipids, by modulating the enzymes involved in cholesterol metabolism, and by reducing oxidative stress and the antagonistic properties of platelet aggregation. Soluble fibres within the linseed are potentially effective in regulating plasma levels of TC and LDL-C. In previous studies ${ }^{(17,18)}$, both in human subjects and in animals, the action of lipid-lowering soluble fibres has been reported. The Third Report of the National Cholesterol Education Program ${ }^{(19)}$ explains that the regular consumption of fibre $(20-30 \mathrm{~g} / \mathrm{d})$ and $5-10 \mathrm{~g}$ of soluble fraction reduces the absorption of saturated fats and cholesterol by up to $7 \%$. When analysing the plasma concentration of HDL-C, the groups did not differ significantly. After the experimental period, the levels of HDL-C are increased in GI and GII. Some previous studies ${ }^{(11)}$ have indicated that, in the presence of linseed, the serum concentration of HDL-C may increase or decrease. These differences may be associated with the concentration of cholesterol present in the diet and the duration of the hypercholesterolaemic diet ${ }^{(11)}$. The quantity of linseed used in this experimental protocol was not effective to promote the reduction of TAG in the GII animals. Previous animal studies ${ }^{(12)}$ have reported different effects of linseed on the concentration of TAG. These inconsistent results could be secondary to different dosages, the type and amount of linseed, as well as by the animal model used $^{(20)}$. However, to date, there is no scientific literature with a plausible explanation for the rise in TAG levels; therefore, it requires future investigations to clarify these mechanisms ${ }^{(21,22)}$. Lee \& $\operatorname{Prasad}^{(23)}$ demonstrated that hypercholesterolaemic diets produce intimal thickness associated with foam cells. These findings are also present in the present study. By morphometric analysis, we observed that all the rabbits developed advanced atherosclerotic lesions (type III) in the aortic arch and descending aorta, which indicates that the dietary model used is extremely effective in the atherogenic process. Lucas et al. ${ }^{(24)}$ reported that the intake of linseed can reduce the formation of atherosclerotic lesions by $50 \%$, whereas the lignan proves to be more effective, as it has a reducing effect of $75 \%$. In a similar study, $\operatorname{Prasad}^{(9)}$ examined the anti-atherogenic activity of linseed in different groups of rabbits. The animals were subjected to different treatments and a hypercholesterolaemic diet was induced in some groups with the supplemental addition of $1 \%$ cholesterol. At the end of the study, the authors concluded that the daily supply of linseed at a dose of $7.5 \mathrm{~g} / \mathrm{kg}$ body weight

Table 2. Median, minimum and maximum cholesterol values (Mean values and standard deviations, $n$ 10)

\begin{tabular}{|c|c|c|c|c|c|c|}
\hline TC & Group & Mean $(\mathrm{mg} / \mathrm{l})$ & Mínimum (mg/l) & Maximum (mg/l) & SD & $P$ \\
\hline \multirow[t]{2}{*}{ TC initial } & GI & $703 \cdot 0$ & $470 \cdot 0$ & $1150 \cdot 0$ & $21 \cdot 79$ & \\
\hline & Gll & $768 \cdot 0$ & $550 \cdot 0$ & $910 \cdot 0$ & $12 \cdot 07$ & $0.420^{\star}$ \\
\hline \multirow[t]{2}{*}{ TC final } & GI & $16767 \cdot 0$ & $3470 \cdot 0$ & $28850 \cdot 0$ & 768.67 & \\
\hline & GII & $10068 \cdot 3$ & $12 \cdot 5$ & $23130 \cdot 0$ & $638 \cdot 60$ & $0.046 \dagger$ \\
\hline \multirow[t]{2}{*}{ TC variation (\%) } & GI & 23792.9 & $6081 \cdot 6$ & 37986.5 & $1204 \cdot 08$ & \\
\hline & GII & $12497 \cdot 7$ & -984.9 & $24417 \cdot 6$ & $808 \cdot 62$ & $0.024^{*}$ \\
\hline
\end{tabular}

TC, total cholesterol; GI, group 1; GII, group II.

${ }^{*}$ Mean values were significantly different $(P<0.05$ by Student's $t$ test).

$\dagger$ Mean values were significantly different $(P<0.05$ by ANCOVA; TC initial as covariable). 
Table 3. LDL-cholesterol (LDL-C) values

(Mean values and standard deviations, $n$ 10)

\begin{tabular}{llrrr}
\hline LDL-C & Group & Mean $(\mathrm{mg} / \mathrm{l})$ & \multicolumn{1}{c}{ SD } & \multicolumn{1}{c}{$P$} \\
\hline LDL initial & GI & 118.0 & 15.17 & \\
& GII & 301.0 & 13.20 & $0.005^{\star}$ \\
LDL final & Gl & 15961.2 & 743.03 & \\
& GII & 10743.2 & 520.66 & $0.108 \dagger$ \\
LDL variation (\%) & Gl & 383069.5 & 35627.36 & \\
& GII & 40935.5 & 2098.81 & $0.001^{*}$ \\
\hline
\end{tabular}

GI, group 1; GII, group II.

* Mean values were significantly different $(P<0.05$ by Student's $t$ test).

† Mean values were significantly different $(P<0.05$ by ANCOVA; initial LDL-C as covariable).

for a duration of 8 weeks was responsible for reducing the development of atherosclerosis by $69 \%$. In a comparative analysis of the existence and extent of atherosclerotic lesions in the animals, we concluded that the consumption of linseed was not effective in reducing the atherogenicity, but the time to exposure to linseed was only $27 \mathrm{~d}$. Histological sections of the aortic arch and descending aorta showed similarities in the thickness of the intima and the intima:media layer area ratio, a result that we do not expect with the anti-atherogenic activity of linseed. There are several hypotheses to explain this contradictory event. Rouleau et $a{ }^{(25)}$ reported that the severity of atheromatous lesions is directly associated with the degree of hypercholesterolaemic diet. It is noteworthy that the atherogenic diet used in this experiment developed extremely advanced lesions, and given this fact, it is difficult for any food with functional properties, such as linseed in the administered dosage, to have anti-atherogenic effects. In addition, Dupasquier et al. ${ }^{(26)}$ concluded that hypercholesterolaemic diets for long periods, such as 8 weeks, limit the anti-atherogenic activity of linseed. In addition, in the studies cited above, the authors used the active substance lignan that probably is more effective than ground flaxseed. Finally, it is known that the aetiology of atherosclerosis is associated with and mediated by inflammatory processes ${ }^{(25,26)}$. This statement is consistent with the present findings, as daily administration of a hypercholesterolaemic diet triggered the formation of atherosclerotic lesions associated with the presence of inflammation. Both experimental groups showed significant positive areas for vascular cell adhesion molecule- 1 and intercellular adhesion molecule- 1 in the intima of the aortic segments. However, the high concentration of soluble cell adhesion molecules in the group of animals that were supplemented with linseed was unexpected. Linseed has a high concentration of $n-3$ fatty acids, which confers anti-inflammatory properties. Many studies have shown an inverse correlation between the concentration of $n-3$ fatty acids, the inflammatory process and endothelial dysfunction. It is believed that the PUFA is responsible for the reduction in the expression of inflammatory markers including the adhesion molecules, vascular cell adhesion molecule- 1 and intercellular adhesion molecule- ${ }^{(15)}$. These conflicting results suggest that the amount and duration of the hypercholesterolaemic diet can influence the effect of $n-3$ fatty acid.

\section{Study limitations}

Although the amount of linseed used in the present study was sufficient to interfere in the lipid profiles of the rabbit, it did not affect atherosclerotic lesions. A previous study from our laboratory demonstrated that a diet comprised of $1 \%$ cholesterol from lyophilised egg induced advanced atherosclerotic lesions (types III, IV and V) in rabbits within 4 weeks (RS Matos, LAV Baroncini, LB Précoma, G Winter, PHL Caron, F Kaiber and DB Précoma, unpublished results). The atherosclerotic lesions induced in this study probably were too advanced for interference by any functional foods over a short study period. Previous studies that examined the effects of linseed administered the bioactive substance, lignan, whereas we administered ground flaxseed. This could contribute to discrepancies between previous studies and the present results. In addition, we did not interfere in the satiation of the rabbits, which could explain their elevated cholesterol levels. Also, we did not weigh tissues or measure liver cholesterol or TAG. The experimental design of the present study did not incorporate a third group of animals administered linseed from day 1 of the experiment. Such a group could have been used to assess the potential preventive effect of linseed consumption. In summary, we acknowledge certain methodological limitations, such as the extent of the hypercholesterolaemic diet, the amount of linseed used and the short study duration, that might have contributed to the results obtained in the present study.

\section{Conclusions}

Linseed consumption exhibited lipid-lowering action by reducing serum TC and LDL-C during a short period of time. The experimental model used in the present study is effective in causing atherosclerosis. In addition, the extent and type of atherosclerotic lesions were directly proportional to the concentration of plasma cholesterol, which increased with the percentage of cholesterol in the diet. Through histological, morphometric and immunohistochemical analysis, linseed had no significant effect on the presence and extent of atherosclerotic lesions in animals administered with a hypercholesterolaemic diet (1\% cholesterol) for a short period of time.

\section{Acknowledgements}

This study was conducted with no financial support or external funding. D. B. P., L. A. V. B. and C. R. P. designed the study. L. A. V. B. also wrote the text. L. B. P., P. H. L. C., G. W., and M. O. D. P. handled the animals and helped to prepare the slices for histological analysis. All authors read and approved the final text. The authors declare that there are no conflicts of interest in this paper.

\section{References}

1. Delmas D, Jannin B \& Latruffe N (2005) Resveratrol: preventing properties against vascular alterations and ageing. $\mathrm{Mol}$ Food Res 49, 377-395. 
2. Fornés NE, Martins IS, Velásquez-Meléndez G, et al. (2002) Escores de consumo alimentar e níveis lipêmicos em população de São Paulo, Brasil (Food consumption scores and serum lipids levels in the population of Sao Paulo, Brazil). Rev Saúde Pública 36, 12-18.

3. Kris-Etherton PM, Harris WS \& Appel LJ (2003) Omega-3 fatty acids and cardiovascular disease: new recommendations from the American Heart Association. Arterioscler Thromb Vasc Biol 23, 151-152.

4. Leanne TB, Philippe O \& Szapary MD (2004) Flaxseed and cardiovascular risk. Nutr Rev 62, 18-27.

5. Prasad K (1997) Dietary flax seed in prevention of hypercholesterolemic atherosclerosis. Atherosclerosis 132, 69-76.

6. Prasad K (2005) Hypocholesterolemic and antiatherosclerotic effect of flaxseed. Atherosclerosis 179, 269-275.

7. Bhathena SJ, Ali AA, Haudenschild C, et al. (2003) Dietary flaxseed mela is more protective than soy protein concentrate against hypertriglyceridemia and steatosis of the liver in an animal model of obesity. J Am Coll Nutr 22, 157-164.

8. Stary HC, Chandler AB, Dinsmore RE, et al. (1994) A definition of advanced types of atherosclerotic lesions and a histological classification of atherosclerosis. A report from the Committee on Vascular Lesions of the Council on Arteriosclerosis, American Heart Association. Circulation 92, $1355-1374$.

9. Prasad K (2004) Antihypertensive activity of secoisolariciresinol diglucoside isolated from flaxseed: role of guanylate cyclase. Int J Angiol 13, 7-14.

10. Klag MJ, Ford DE, Mead LA, et al. (1993) Serum cholesterol in young men and subsequent cardiovascular disease. $N$ Engl $J$ Med 328, 313-318

11. Prasad K (2005) Hypochelesterolemic and antiatherosclerotic effect of flax lihnan complex isolated from flaxseed. Atherosclerosis 179, 269-275.

12. Cunanne SC, Hamadeh MJ, Liede AC, et al. (1995) Nutritional attributes of traditional flaxseed in healthy young adults. $\mathrm{Am}$ J Clin Nutr 61, 62-68.

13. Shakir KAF (2007) Madhusudhan B hypocholesterolemic and hepatoprotective effects of flaxseed chutney: evidence from animal studies. Ind J Clin Biochem 22, 117-121.

14. Dodin S, Cunnane SC, Mâsse B, et al. (2008) Flaxseed on cardiovascular disease marker in healthy menopausal women: a randomized, double-blind, placebo-controlled trial. Nutrition 24, 23-30.

15. Kitts DD, Yuan YV, Wijewickreme AN, et al. (1999) Antioxidant activity of the flaxseed lignan secoisolariciresinol diglycoside and its mammalian lignans metabolites enterodiol and enterolactone. Mol Cell Biochem 202, 91-100.

16. Foti M, Piatelli M \& Baratta MT (1996) Flavonoids, coumatism, and cianamic acids as antioxidants in micellar system: structure activity relationship. I Agric Food Chem 44, 497-501.

17. Ruberfroid M (1993) Dietary fiber, inulin and oligofrutose: a review comparing their physiological effects. Crit Rev Food Sci Nutr 33, 1303-1348.

18. Sawashita N, Naemura A, Shimizu M, et al. (2006) Effect of dietary vegetable and animal proteins on atherothrombosis in mice. Nutrition 22, 661-667.

19. Pereira MA, O'Reilly E, Augustsson K, et al. (2004) Dietary fiber and risk of coronary heart disease: a pooled analysis of cohort studies. Arch Intern Med 164, 370-376.

20. Babu US, Mitchel GV, Wiesenfeld P, et al. (2000) Nutritional and hematological impact of dietary flaxseed and defatted flaxseed meal in rats. Int J Food Sci Nutr 109-117.

21. Zagarra IGE \& Schwarz EE (2006) Impact of dietary patterns and intervensions on cardiovascular health. Circulation 114, 961-973.

22. Caterina R, Liao JK \& Libby P (2000) Fatty acid modulation of endothelia activation. Am J Clin Nutr 7, Suppl. 1, 213S-223S.

23. Lee P \& Prasad K (2003) Suppression of oxidative stress as a mechanism of reduction of hypercholesterolemic atherosclerosis by cyclooxygenase inhibitors. Int J Angiol 12, $13-23$.

24. Lucas EA, Lightfoot SA, Hammond LJ, et al. (2004) Flaxseed redeuces plasma cholesterol and atherosclerotic lesion formation in ovariectomized Golden Syrian Hamsters. Atherosclerosis 173, 223-229.

25. Rouleau JL, Parmkely WW, Stevens J, et al. (1983) Verapamil suppresses atherosclerosis in cholesterol fed rabbits. J Am Coll Cardiol 1, 1453-1460.

26. Dupasquier CH, Weber AM, Ander BP, et al. (2006) Effects of dietary flaxseed on vascular contractile function and atherosclerosis during prolonged hypercholesterolemia in rabbits. Am J Physiol Heart Circ Physiol 291, H2987-H2996. 